\title{
UEBER DEN BAUINSTINKT EINER KÖCHERLARVE (LIMNOPHILUS MARMORATUS CURT.)
}

VON

\author{
J. A. BIERENS DE HAAN \\ A M S TERD A M.
}

Die Frage nach das Wesen der tierischen Instinkte und ihrer Äusserungen ist wohl ohne Zweifel das Kernproblem der jungen Wissenschaft, die sich das Studium der tierischen Psyche zum Ziel gesetzt hat. Obwohl dieses schon mehrmals gesagt ist, und in allgemeinen auch wohl zugegeben wird, gibt es doch nur wenige Untersuchungen, die direkt auf dieses Problem gerichtet sind. Die Arbeiten auf tierpsychologischem Gebiete, so fern sie nicht in das Grenzgebiet der Physiologie der Sinnesorgane fallen, betreffen bei den niederen Tieren meistens das Lernvermögen und die Gewohnheitsbildungen, bei den höheren dazu noch die Intelligenzäusserungen, Fragen die natürlich äusserst wichtig sind, aber doch den Kern der tierischen Psyche nur in so weit berühren, als sowohl Intelligenzhandlung als. Gewohnheitsbildung auf dem Boden des instinktieven Trieblebens aufgebaut sein muss. Nur die Arbeiten über die sogenannten Tropismen und verwandte Erscheinungen, besonders in so weit sie eine mechanistische Erklärung derselben ablehnen, und die Tropismen als durch äussere Faktoren (z. B. Licht oder Schwere) gerichtete Instinkthandlungen auffassen, können in den Rahmen der Instinktstudien aufgenommen werden, während die vielen Beobachtungen im freien Felde, die von einem Beobachter wie FABRE bis zum einfachen Schulbuben, der "Natursport" treibt, hinabreichen, uns zwar ein ungeheures Material über Instinktäusserungen geliefert haben, meistens aber $\mathrm{zu}$ weinig wissenschaftlich aufgefasst sind, und $\mathrm{zu}$ viel dem Zufall überlassen, um für exakte Instinktuntersuchungen viel Brauchbares liefern zu können.

Eine Erklärung für diesen Mangel an solchen Untersuchungen ist nicht schwer zu geben. Zur natürlichen Entfaltung ihrer Instinkte bedürfen die Tiere einer für sie natürlichen Umgebung, die im Laboratorium meistens nicht leicht nachzuahmen ist. Die Beobachtungen im Felde dagegen erfordern viel Zeit und Glück, und das dabei so komplizierte Milieu eignet sich meistens wenig für Experimente. Und dazu sind die bekanntsten und anregendsten Instinkthandlungen der Insekten und Vögel zu kompliziert und auch zu spezialisiert, um noch Hoffnung zu geben, dass man durch experimenteller Eingriff viel an ihrem Ablauf änderen könnte, was doch für ein tieferes Verständnis derselben nötig wäre.

Aber es gibt doch ein Milieu, das im Laboratorium relativ leicht, wenn auch vielleicht nicht ganz dem normalen ähnlich nachzuahmen ist, nämlich das der in Teichen und Tümplen lebenden kleineren Wassertiere, die sich nicht über grössere Strecken zu bewegen pflegen. Durch Aquarien und Behälter mit lebenden Pflanzen und frischem Wasser kann man dieses einfaches Milieu ziemlich gut herstellen, so dass die Tiere sich leicht wie zu Hause fühlen, und entsprechend ihrer einfachen Umwelt zeigen solche Tieren meistens auch keine sehr verwickelten Instinktäusserungen.

Am konkretesten liegen die Resultate der Instinkthandlungen vor uns bei den mehr oder weniger verwickelten Bauten, die einige Tiere herstellen. Wenn man sonst den Ablauf einer Instinkthandlung nur beobachten und beschreiben kann, so liegen hier die materiellen Resultate vor uns, die 
man in der Hand nehmen, messen, wägen, zeichnen, photographieren kann. So scheint es mir denn ratsam, dass derjenige, der die Instinkte studieren will, mit solchen konkreten Ergebnissen anfänge, und weil die Vögel sich im allgemeinen weniger dazu eignen, scheinen mir die bauenden Insekten und besonders die Wasserinsekten das beste Material zu sein, wenn man einen Instinkt in seinen Variationen, in seiner Breite und Schärfe und Beeinflussung durch experimentellen Eingriff studieren will.

Und bei jeder Studierung dieser noch so rätselhaften Triebe, die man die Instinkte der Tiere nennt, soll man sich immer vor Augen halten die grosse Verwandtschaft ihrer Äusserungen mit dem, was uns als ontogenetische Entwicklung bekannt ist, eine Verwandtschaft, auf welche schon öfters von Biologen und Philosophen (Schopenhauer, Bergson) hingewiesen ist, und die soweit geht, dass man bisweilen nicht sagen kann, ob eine sich abspielende Änderung als Instinkthandlung oder besser als morphogenetischer Vorgang zu betrachten ist. Zeigt diese Uebereinstimmung vielleicht, dass man sich hier einer gemeinsamen Wurzel nähert, auf welcher die reiche Mannigfaltigkeit des tierischen Lebens und Treibens aufgebaut ist?

$\mathrm{Zu}$ solchen Fragen sẹ die hier gebotene Untersuchung über den Bauinstinkt einer einfachen Köcherlarve ein sehr bescheidener Beitrag.

Das Material, mit dem ich während einiger Frühlingsmonate arbeitete, waren die Larven des Trichopterons Limnophiltus marmoratus, die in grossen Mengen in einem Teiche im Walde am Füsse des SALÈve's, unweit GENF, vorkamen. Der Teich war ganz von Fichten und kleinen Eichen umgeben, der sandige Boden war mit halbvermoderten Fichtennadeln, Eichenblättern und Ästchen bedeckt. Aus diesem Material hatten sich die Larven ihre bekannten, 1 bis $3 \mathrm{~cm}$ langen Köcher gebaut, ungefähr von zylindrischer Form, wobei das Vorderende immer etwas breiter war als das Hinterende, das von einer Membran mehr oder weniger abgeschlossen war. Wenn die Tiere älter und grösser waren, wurde auch gröberes Material benutzt, bei kleineren Köchern waren die Fichtennadeln nicht grösser als $5 \mathrm{~mm}$, bei grösseren fand man Fragmente bis $1,5 \mathrm{~cm}$, auch wohl dickere Ästchen und Borkenstücken von einigen Zentimetern Länge und Breite. Nur einmal auf einige hunderte Köcher, die ich in der Hand bekam, fand ich auch Konchilien als Material benutzt (Planorbis und kleine Bivalven). Durch ihr Material hatten die Köcher ungefähr das spezifische Gewicht des Wassers, wodurch die Larven instande waren sich durch kräftige Bewegungen ihres aus dem Köcher gestreckten Vörderleibes durch das Wasser fortzubewegen, und nach der Oberfläche des Wassers zu steigen oder zu sinken. Das Material der Köcher ist immer senkrecht zur Körperachse angelegt, die Köcher gehören also zur 3. Klasse nach der STRUCK'schen Einteilung ${ }^{1}$ ).

Die Erste Frage, die sich stellen lässt, ist nun die folgende: Was machen die Larven, wenn man sie aus ihrem Köcher vertreibt? (dieses gescheht sehr leicht und ohne das Tier zu schädigen, wenn man es von hinten z. B. mit einer Fichtennadel reizt, oder den Köcher vorsichtig abbricht). Ist der Bauinstinkt stark genug und genügend angepasst um sie unter diesen abnormalen Umständeñ zu neuem Bau zu bringen?. (denn normalerweise baut die Larve langsam während ihres Wachstums weiter, und ein freiwilliges oder gezwungenes Verlassen des Köchers wird wohl selten oder nie vorkommen) Aus Versuchen von PicteT ${ }^{2}$ ), Ostwald ${ }^{3}$ ), Marshall und Vorhies ${ }^{4}$ ), Wesenberg Lund ${ }^{5}$ ) und anderen ist bekannt, das dies in der Tat bei den meisten Tieren der Fall ist, obwohl auch die entgegengesetzten Data vorliegen ${ }^{6}$ ). Limnophilus marmoratus macht jedenfalls einen Neubau, und dieses geschieht wie folgt.

Wenn man die aus ihrem Köcher getriebene Larve in einen kleinen Behälter mit Wasser bringt,

1) R. STRUCK. Neue und alte Trichopterenlarvengehäuse. Ill. Zeitschr. für Entomologie IV 1899.

2) F. J. PICTET. Recherches pour servir à l'histoire et à l'anatomie des Phryganides. Genève, 1834.

3) Wo. Ostwald. Experimental-Untersuchungen über den Köcherbau der Phryganidenlarven. Zeitschr. für Naturwiss. 72, 1889.

- - Ueber die Variabilität der Gehäuse der Trichopterenlarven. Zeitschr. für Naturwiss. 74, 1901.

4) W. S. MARShALL and C. T. VorhIES. The repair and rebuilding of the larval case of Platyphylax designatus Walk. Biol. Bull. IX 1905.

5) C. Wesenberg Lund. Ueber die Biologie des Phryganea grandis und über die Mechanik ihres Gehäusebaues. Int. Rev. ges. Hydrob. und Hydogr. IV 1911.

6) R. STRUCK. Beiträge zur Kenntnis der Trichopterenlarven. Mitt. Geogr. Ges. und Nat. Mus. Lübeck XVII, 1903. 
sieht man, wie das vorher so ruhige Tier sich lebhaft $z$ u bewegen anfängt und ziemlich schell im Behälter umherkriecht. Diese Bewegung kann in einem Behälter ohne Material oder mit unbenutzbarem Material tagelang daueren. Diese allgemeine Unruhe geht aber in zweckmässige Aktivität über, sobald man dem Tiere normales Baumaterial, in casu etwas halb vermoderte Fichtennadeln reicht. Mit Extremitäten und Mundteilen fängt es dann an, die Materialteilchen zusammenzubringen, wobei durch Schleimfäden das Material zusammengeklebt wird. Dan fängt es an, durch drehende Bewegungen um seine Körperachse und wellenformige Bewegungen des Leibes sich in dieses klebende Material einzurollen. Das Resultat davon ist, dass es nach etwa einer Stunde eingewickelt ist in einer Menge mehr oder weniger fest zusammenhangender Fichtennadeln, die aber nicht so fest zusammenhangen dass sie unbeweglich wären, sodass durch die fortschreitenden Bewegungen des Tieres durch das Wasser sie sich mehr oder weniger parallel dem Körperachse verschieben und wie eine Schleppe dem Tiere anhangen. Bei diesem vorläufigen Köcher wird das Material als ganzes benutzt, also nicht durchgebissen, was man am leichtesten sehen kann, wenn man dem Tiere nur Material von gewisser Länge, z. B. ganze Fichtennadeln darbietet.

Allmählich geht dieser Prozess in einem zweiten über, wobei der eigentliche Köcherbau stattfindet. Jetzt werden nicht mehr ganze Nädeln angeklebt, sondern diese werden erst mit den Vorderbeinen gegen dem Körper gedrückt, und zu einer Länge, die dem Abstand zwischen Mund und Ende des Vörderbeines ungefähr entspricht, abgenagt; dann werden die abgebissen Stücke regelmässig quer zur Körperachse niedergelegt und angeheftet. So baut die Larve an der Vörderseite regelmässig weiter, bis endlich der vorläufige Köcher nur noch als ein Anhang am Wohnköcher mitgeschleppt wird, und er schliesslich abbricht und die Larve in ihrer neuen Wohnung fortkriecht. Der ganze Prozess ist in etwa 4 Stunden abgelaufen, die neue Wohnung ist meistens nur etwas kleiner und feiner, sonst aber der alten gleich.

Die Frage ist jetzt: wie gross ist die Instinktbreite? d. h. welche Materialien werden von den Tieren zum Köcherbau benutzt? Hierüber liegen schon Versuche von Ostwald vor (1899 und 1901), der mit verschiedenen Arten arbeitete, und fand, dass fast jedes Material zum Bau benutzt wurde. So einfach ist die Sache doch nicht, jedenfalls nicht bei L. marmoratus. Wie gesagt, waren Fichtennadeln das normale Baumaterial, mit Kiefernadeln dagegen wussten sie nichts zu erreichen. Erst habe ich ihnen $4 \mathrm{~cm}$. lange Nadeln einer Zierkiefer aus einer Parkanlage gegeben. Nach 20 Stunden hatten die Tiere selbst noch keinen vorläufigen Köcher gebaut, und erst als.ich ihnen dann etwas normales Material unter die Kiefernadeln mischte, wurde mit diesem der Bau angefangen. Auch wenn ich die Kiefernadeln zerschnitt, und den Tieren Stücke von 2 oder $1 \mathrm{~cm}$. oder ganz fein zerschnittene reichte, wurde damit nicht gebaut; nur das Tier dem die $1 \mathrm{~cm}$ langen Stücken gegeben waren, baute einen vorläufigen Köcher, aus welchem es nachher wieder hinauskroch. $\mathrm{Zu}$ grosse Härte des Materiales, wodurch die Nadeln nicht auf gewünschte Grösse abgebissen werden könnten, war also nicht die Ursache des Misslingens. Mit Nadeln der gewöhnlichen Kiefer wurden übrigens ebensowenig Resultate erreicht und auch hier trat wie bei den Nadeln der Zierkiefer relativ schnell der Tod der Tiere ein. Man könnte meinen, dass dieser Tod die Folge irgendeines in den Nadeln befindlichen chemischen Substanzes wäre, wahrscheinlich ist dies aber nicht, da immer beim Nichtgelingen eines Baues die Tiere schnell eingehen, vielleicht durch $\mathrm{zu}$ grosse Anstrengung.

Aber sogar die Nadeln der Fichte, ihr normales Material, werden nicht immer zum Bau verwendet. So versuchte ich es einmal: $a$. mit jungen, frischen, hellgrünen Fichtennadeln, $b$. mit alten, dunkelgrünen, beide vom Baum gepflückt, $c$. mit trockenen, abgefallenen, $d$. mit aus dem Wasser genommenen, etwas vermoderten Fichtennadeln. Während $d$. in etwa 4 Stunden einen Köcher baute, und $c$. dasselbe in einer Nacht zustande brachte, gingen die Tiere bei $a$ und $b$ nach einigen Tage ein, ohne selbst einen vorläufigen Köcher gebaut zu haben. Aus Material wie $d$, dass ich zuvor ganz fein zerschnitten hatte, wurde in normaler Zeit ein Köcher gebaut, vielleicht selbst etwas schneller wie sonst, weil das Zernagen der Nadeln jetzt nicht nötig war.

Verschiedene anderen Materialien wurden noch versucht. Dass Messingspäne (1 cm lang) und Messingpulver kein Resultat ergaben, wunderte mich weniger, als dass sie auch aus dem weissen Holz von Streichhölzern, selbst wenn dieses in kleine Stückchen gespalten war, nicht zu bauen wussten. Aus Splittern von Zimmerholz wurde, obwohl etwas verlangsamt, ein etwas unregelmässiger Köcher 
gebaut. Von fein zerschnittenen Stückchen von Kiefer- und Fichtenzapfen wurden normale Köcher gebaut, aber die Tiere gingen nachher bald ein. Aus Stückchen zerschnittener Eichenborke, die ich einige Zeit im Wasser hatte liegen lassen, wurden relativ normale Köcher in kurzer Zeit verfertigt; Moos, Teile von grünen Wasserpflanzen, selbst Brot wurden zum Bau vorläufiger Köcher verwendet.

Es zeigt sich also, dass die Instinktbreite gering ist, m.a.W. das nur wenige und nur dem normalen ähnliche Materialien zum Bau verwendet werden, und werden können, ohne die Tiere zu schädigen. In letzter Instanz liegt die Ursache dieser Verschiedenheiten natürlich in dem chemischen oder physikalischen Zustand der gebotenen Materialien, denn der Trieb, sich eine schützende Hülle $z \mathfrak{u}$ bauen, blieb bei jedem Material lebhaft, und zeigte sich erfolgreich, sobald die Larven nach dem Misslingen ihrer Versuche in normales Material zurückgebracht wurden. Der Fehler lag also daran, dass obwohl der Trieb genügend stark blieb, die aus dem Trieb entspringende Handlung so spezialisiert war, dass kleine Schwierigkeiten mit etwas vom normalen abweichenden Material nicht mehr überwunden werden konnten (die Tiere versuchten es mit jedem Material das man ihnen gab). Der Trieb war also stark genug, die Handlung aber zu eng zu bestimmten Bewegungen beschränkt und nicht mehr genügend plastisch.

Ein wichtiges Versuchsmaterial war S a n d, weil bekanntlich daraus viele Trichopterenlarven bauen, und nach STRUCK auch Sandköcher von L. marmoratus in der Natur wohl vorzukommen scheinen. Bauen auch die Fichtennadel-Tiere einen Köcher aus feineren oder gröberen Sand, nachdem man sie aus ihrem eigenen verjagt hat? Hier zeigte sich eine Merkwürdigkeit, die bei anderm Material weniger deutlich war, nämlich dass der Bautrieb zu unterscheiden ist von einem wohl älteren und mehr allgemeinen Trieb, sich den unbeschützten Körper zu bedecken. Wenn man die Tiere ihres Köchers beraubte und sie in eine Behälter mit Sand setzte, krochen sie darin fortwährend umher, alsob sie irgend etwas suchten um damit den Bedeckungstrieb zu befriedigen, wozu der Sand sich schlecht zu eignen schien. Gab man ihnen dann z. B. etwas Moosblätter, aus welche sie eine primitieve Bedeckung machen konnten, und nam dann die übrigen Moosblätter fort, dann bauten sie an dieser sofort einen Sandköcher normaler Grösse an. Fanden sie nichts, dann dauerte es einige Tage bevor sie, meistens bei Nacht, sich entschliessen konnten, sich ganz in Sand einzuhüllen und damit weiter zu bauen ${ }^{1}$ ). Auch andere Objekte genügten zur Befriedigung dieses Schütztriebes, z. B. kleine Gummiringe. Wenn man ihnen einen selbst nur $2 \mathrm{~mm}$ grossen Ring, aus einem Gummischlauch geschnitten, in ihrem Behälter gibt, kriechen sie hinein und fangen sofort an, mit Sand an dem Ring weiter zu bauen, der nachher, wie der vorläufige Köcher bei dem normalen Bau abgeworfen wird. Der Ring der nur 1/5-1/10 des Körpers bedeckt, genügt also den Bedeckungstrieb zu befriedigen.

Derselbe Bedeckungstrieb wird also auch wohl Anlass sein zum ersten Akte des Baus aus normalen Material, der Einwicklung in den vorläufigen Köcher, die dem eigentlichen Bau vorhergeht. Der Trieb, sich aus bestimmten Materialien charakteristische Köcher zu bauen (früher meinte man dass die Köcher so spezialisiert waren, dass man die Arten daran determinieren könnte) ist dann als Spezialisierung eines mehr algemeinen Triebes zu betrachten, sich den nackten Körper zu schützen. Der Bedeckungstrieb ist wohl genetisch älter und dabei weniger spezialisiert (cum grano salis könnte man den einen Trieb mit einer Genus-eigenschaft, den anderen mit einer Spezies-eigenschaft vergleichen), diese beiden Eigenschaften findet man wieder zurück in der Tatsache dass vor Anfang des eigentlichen Baues erst dieser Schütztrieb befriedigt sein muss, und dass der Schütztrieb mehr allgemein $z u$ befriedigen ist als der Bautrieb.

Dass der Bedeckungstrieb mehr allgemeiner Art ist, und weniger spezialisierten Bahnen folgt, zeigt sich aus der Leichtigkeit, womit verschiedene Gegenstände als Wohnraum benutzt werden. Die ihres Köchers beraubte Larve zieht in jeden Gegenstand hinein, in welchem ihr Körper passt. Nicht nur ihre eigene Wohnung oder die eines Artgenossen wird benutzt (dass sie dabei keine Vorliebe für ihren eigenen Köcher vor einem fremden zeigt, beweist dass individuelle Unterschiede, z. B. ein individueller Duft, fehlt), sondern auch Gummiröhrchen von verschiedener Länge werden akzeptiert, sowie Glasröhrchen oder ausgehöhlten Holzstücke, die etwas beschwert und zum Sinken gebracht waren.

1) Auch OsTWALD (1901) fand, dass für Sandbau bei L. marmoratus ein Vorbau aus anderen Materialien beschleunigend wirkte. 
Waren diese Wohnungen nun etwa ebenso gross wie die eigener Köcher, dann lebten die Tiere darin ruhig weiter; waren die gegebenen Röhren kleiner, z. B. 5 bis $7 \mathrm{~mm}$, dann wurde der Bauinstinkt wieder angeregt, und an der dargebotenen Wohnung eine neue aus mitgegebenem Baumaterial angebaut.

Und hierbei zeigte sich eine Merkwürdigkeit die mir wichtig zu sein scheint, nämlich die gradweise Vorliebe für bestimmte Materialien, die auch zu vorschein kam bei Reparaturversuche, die ich bei Tieren in verschiedenen Köchern anstellte.

Bei solchen R e para turversuch e n wurde dem Tiere der vordere oder hintere Teil seines Köchers abgenommen, um zu sehen, ob überhaupt, und wenn ja mit welchem Material, wieder angebaut wurde. Nebenbei sei gesagt, dass es keinen Unterschied machte, ob man das Vorderende oder das Hinterende des. Köchers abnam, in beiden Fällen kroch das Tier zuerst in dem restierenden Teil seines Köchers zusammen, und baute dann von dort aus an der Vorderseite weiter. Ein Tier dem nun der Köcher bis auf $1 \mathrm{~cm}$ abgenommen war, und das dann in einen Behälter mit Sand gebracht wurde, reparierte seinen Köcher nicht, auch nicht wenn ich am nächsten Tag ihn noch einmal eines Teiles seines Köcherrestes beraubte. Wenn es dann nach 2 Tagen noch nichts angebaut hatte, gab ich ihm etwas normales Material, womit er sofort $\mathrm{zu}$ bauen anfing, sodass nach einigen Stunden sein Köcher wieder $15 \mathrm{~mm}$ lang war, wobei mann deutlich die angebauten $7 \mathrm{~mm}$ von der $8 \mathrm{~mm}$ langen Reste seines ursprünglichen Köchers unterscheiden konnte. Wenn es dann nach einigen Tagen an den dann $20 \mathrm{~mm}$ langen Köcher nicht mehr anbaute, entfernte ich das Neugebaute und ein Teil des ursprünglichen Köchers noch einmal, und brachte es wieder in einen Behälter mit Sand zurück, aber das Tier, das doch bewiesen hatte zur Reparatur seines Köchers imstande zu sein, war wiederum in 3 Tagen nicht zur Reparatur mit Sand zu bringen, tat dieses aber flott, sobald ich ihm wiederum normales Material zur Verarbeitung gab. In einigen Tagen hatte es wiederum seinen Köcher bis auf $18 \mathrm{~mm}$ verlängert.

Dass die Tiere nicht imstande sein solten mit Sand zu reparieren wäre aber ein falscher Schluss. Tiere die man dazu gebracht hat einen Sandköcher zu bauen, reparieren diesen nämlich wohl mit Sand. Eines von diesen welches eines $15 \mathrm{~mm}$ langen Köcher aus groben Sand gebaut hatte, wurde des Hinterendes seines Köchers bis auf 5 bis $7 \mathrm{~mm}$ beraubt, und dann wieder in Sand gebracht. Am nächsten Tag hatte es am Vorderende schon $8 \mathrm{~mm}$ angebaut. Auch eines, welches einen Köcher von feineren Sande gebaut hatte, reparierte diesen in einer Nacht von 5 auf $15 \mathrm{~mm}$, und baute selbst noch etwas weiter. Auch die Hypothese, dass sie nur mit dem Material reparieren aus welchem ihr Köcher gebaut ist, wäre falsch, denn eine Larve im Sandköcher repariert diesen wohl mit normalem Material. Dazu ist es nicht einmal nötig, erst- den Sandköcher zu verletzen, sondern auch ein Tier, das scheinbar ganz zufrieden in einem Sandköcher lebte, fing sofort an, diesen mit normalen Material auszubauen, wenn ihm dieses in seinem Behälter gegeben wurde. Es war alsob die Fichtennadeln oder vermoderten Eichenblätter in ihm eine Erinnerung an eine bessere Wohnung zurückriefen! Am schönsten zeigte sich diese Vorliebe bei Tieren, die, wie oben beschrieben, in Gummi- oder Glasröhrchen verkrochen waren. Eines welches in ein $1 \mathrm{~cm}$ langes Gummiröhrchen sass, aber dem dieses Röhrchen als Wohnung nicht genügte, baute mit Sand bis $17 \mathrm{~mm}$ an. Als ich ihm dann nachher etwas normales Material dazu gab, baute es sofort damit wieder weiter, und befand sich am nächsten Tag in einer Wohnung, die hinten aus $10 \mathrm{~mm}$ Gummischlauch, in der mitte aus $7 \mathrm{~mm}$ Sand, und vor aus $8 \mathrm{~mm}$ Fichtennadeln bestand. Aber es blieb weiterbauen, und hatte in drei Tagen den Gummischlauch abgeworfen (die Wohnung bestand dann aus $11 \mathrm{~mm}$ normalen Material und $7 \mathrm{~mm}$ Sand). Während einiger Tage blieb dies unverändert, dann brachte ich es in einen Behälter ohne Sand und nur mit normalem Material. Dieses schien sein seinen Bauinstinkt wieder an zu regen, denn in den folgenden Tagen wurde durch weiteren Bau das Verhältnis immer mehr zugunsten des normalen Materiales verschoben, bis es in 12 Tagen in einem ganz aus normalem Baustoff aufgebauten Köcher von $20 \mathrm{~mm}$ lebte, von welchem jetzt auch die letzte Spuren des Sandköchers abgestossen waren.

Solche aus zwei oder mehr verschiedenen Teilen Chimären-artig zusammengestellten Köchern waren in verschiedenen Kombinationen zu bekommen, nur sollte man dafür Sorge tragen, das Material in bestimmter Reihenfolge $z u$ geben. An normalen Köchern wird nicht mit Sand angebaut, bzw repariert, umgekehrt an Sandköchern wohl mit Sand und normalem Material. An Gummiröhrchen u. s. w. baut es sowohl mit Sand als mit normalem Material an. Auch aus Versuchen wobei man die 
Larve nach Entfernung aus ihrem Köcher in gemischtes Material, z. B. Sand und Holzsplitter, oder Holzsplitter und Fichtennadeln bringt, zeigt sich dieselbe Vorliebe. So liesse sich eine ganze Ska la von Materialien zusammenstellen, welche von wenig bevorzugten Materialien bis zu mehr geliebten hinaufsteigt. An deren Spitze stehen dan für L. marmoratus Fichtennadeln, darauf folgen wohl vermoderten Eichenblätter. Beide stehen über feinen und grobem Sand und Eichenborke. Moos steht höher als Sand, und Fichtennadeln höher als Brot, womit auch Köcher angelegt werden; Holzsplitter stehen höher als Sand, und etwas aber nicht viel niedriger als Fichtennadeln, denn ein Köcher von Holzsplittern wird, wenn Fichtennadeln dazu verabreicht werden, mit diesem Material weitergebaut, wobei aber auch ab und $\mathrm{zu}$ ein weisser Holzsplitter verarbeitet wird. Auch wenn man die Larve von Anfang an in gemischtes Material bringt, das aus Holzsplittern und Fichtennadeln besteht, wird der Köcher aus beiden aufgebaut, sei es auch dass die Nadeln die Splitter im Zahl übertreffen.

Ich habe eine solche Skala nicht ausgearbeitet, weil mir das nicht genügend wichtig schien. Wohl aber scheint es mir wichtig, dass sie zusammenzustellen wäre. Noch immer gibt es Personen, welche in den Tieren, besonders den niedrigen, nichts als Reflex-Machinen sehen können $\left.{ }^{1}\right)$. Gäbe es nun allein Materialien die wohl, und solchen die nicht zum Köcherbau anregen, so könnte man auch hier wieder meinen, dass mit der Annahme eines „Baureflexes" alles genügend erklärt sei. Dass aber die verschiedenen Materialien die Tieren in so verschiedener Weise zu Bauhandlungen anregen, dass sie also eine so verschiedene Bedeutung für die Tiere haben, welche von Fall zu Fall wechselt, und dass sie dann auch wohl verschiedene Affekte hervorrufen, dies alles zeigt, dass das psychische Leben dieser einfachen Larven eine grössere Tiefe und Mannigfaltigkeit, man könnte sagen mehr Relief hat, als mit einer Machinentheorie in Übereinstimmung zu bringen wäre ${ }^{2}$ ).

Und für die Instinkte im allgemeinen zeigt es sich, dass diese nicht nur charakterisiert sind durch ihren grösseren oder geringeren $\mathrm{Breite}$, d. h. die grössere oder geringere Zahl und Verschiedenheit der Empfindungen welche die Aktivierung des Triebes hervorrufen, sowie der Handlungen, zu welchen der Trieb Anlass gibt, sondern auch durch ihre Struktur, d. h. den ganzen Zusammenhang von stärker oder schwächer, aktiver oder latenter sein, den der Trieb in seinen Reaktionen auf verschiedenen Wahrnehmungen und Empfindungen zeigt.

1) Neuerdings hat auch wieder DOFLEIN in seinem bekannten Büchlein über den Ameisenlöwen (1916) dieses Tier für einen Reflexautomaten ausgemacht, eigentlich nur auf negativen Grunden.

2) Auch an einem anderen Falle, der nicht direkt mit dem Bauinstinkt verbunden ist, zeigte sich noch, dass die Tiere nicht auf einem so niedrigen psychischen Stadium stehen. Wenn man einer Larve, die aus ihrem Köcher verjagt ist, dieser Köcher wieder vorlegt, kriecht sie wie gesagt nach kurzen Betasten des Köchers wieder hinein. Nun ist aber der Köcher etwas kegelförmig und am Hinterende etwas schmäler als am Vorderende. Das Tier kan dadurch nur am Vorderende hineinkriechen. Folge davon ist, dass es umgekehrt, d.h. mit dem Kopf dem früheren Hinterende zugewandt, in seinem Köcher steckt, und durch die engere hintere Oeffnung den Kopf nicht oder schwierig hinausstecken kann. Das Tier steht nun vor dem Problem: wie bringe ich meinen Kopf hinaus? Ein Reflextier würde auf den Reiz des Sicheingeschlossenfühlens auf eine bestimmte Weise reagieren, z. B. dadurch, dass es aus dem Köcher zurück nach aussen kriecht. Dieses sah ich auch wohl geschehen. Aber damit waren bei den Tiere die Lösungweisen des Problems nicht erschöpft. Anderen fingen an, ihren Köcher an der Hinterseite abzubrechen, einmal sah ich eines mit den Mundteilen Fichtennädelchen losmachen und die Oeffnung dadurch vergrösseren. Gelingt dies in ausreichendem Grade, dann steckt das Tier seinen Kopf durch die Oeffnung, und bleibt dann so umgekehrt zu seiner ursprünglichen Lage sitzen. Meistens aber gelingt dieses ihnen nicht, und dann dreht das Tier sich plötzlich in seinem Köcher um, und sitzt infolgedessen wieder wie zuvor, mit dem Kopf nach der Vorderseite.

Auch hier also zeigt das Tier Initiative, und Wahl zwischen verschiedenen Mitteln ein bestimmtes Ziel zu erreichen. Die Mittel sind ihm angeboren (auch das Umdrehen im Köcher komt wohl mehr ohne für uns verständlichen Anlass vor und ist auch von verschiedenen Untersucher beschrieben) die Benutzung verschiedener Mittel nacheinander zur Erreichung eines bestimmten Zwecks geht aber über die Fähigkeiten eines Reflexautomaten hinaus. Doch wäre es irrig, hierin eine Intelligenzhandlung sehen zu wollen, denn von einen Einsehen der Beziehung zwischen Mittel und Zweck scheint mir hier keine Rede zu sein. Höchstens wird eine solche Beziehung vom Tiere wahrgenommen, was nicht dasselbe ist. Es ist aber, wie es auch CLAPARĖDE für den Trial-and-Error-Bewegungen betont hat ${ }^{3}$ ), eine gewisse Verwandtschaft mit Intelligenzäusserungen nicht zu leugnen. Und dann ist meines Erachtens diese dreifache Lösung des Problems ihres zweckmässigen Charakters wegen höher zu veranschlagen als die echten Versuch-undIrrtum-Bewegungen der niederen Tiere, wobei nur der erfolgreichste aus einer Zahl Bewegungen auf's Geratewohl gewählt und fortgesetzt wird.

Leider fehlte mir die Gelegenheit zu verfolgen, ob noch Erfahrung bei dieser Wahl eine Rolle spielen kann.

3) Ed. ClaPARĖDe. La psychologie de l'intelligence. Scientia, XXII, 1917. 
Wie gesagt, wurden diese Versuche nur mit eine $r$ Spezies von einem Fundort angestellt. Es wäre vielleicht interessant, diese einfachen Versuche an anderen Trichopterenarten oder an Limnophilen vor einem anderen Fundort nachzuprüfen. Interessant wäre es besonders wenn es sich zeigte, dass andere Tiere derselben Art sich in Bezug auf Materialwahl u. s. w. anders verhielten. Meine Versuchtiere entstammten wie gesagt einem kleine Teiche in einem Fichtenwäldchen. In der Umgebung waren keine andere solche Teiche, und in dem einzigen stehenden Wasser, das ich in dieser Gegend noch kannte, einem Tümpel in einer Wiese, habe ich keine Limnophilen finden können. Denkbar wäre also, dass meine Tiere seit mehreren Generationen in diesem einförmigen Milieu gelebt haben. Wichtig wäre es nun, wenn ein Einfluss dieser Umstände auf ihr Verhalten merkbar sein sollte. Von „psychologische Rassen" wissen wir noch nichts, und doch wäre eine Kenntnis solcher Rassen und besonders ihres Entstehen und Vergehen nicht nur für die Tierpsychologie, sondern auch für unser ganzes biologisches Weltbild von grosser Bedeutung. 